\title{
Research Square \\ Identification Of New Sources Of Rice Genotypes (Oryza Sativa L.) Tolerant To Cold Stress
}

Kidist Tolosa Entele ( $\square$ kidisttolosa12@gmail.com )

Makerere University College of agricultural science

\section{Richard Edema}

Makerere University College of agricultural science

\section{Jimmy Lamo}

National Crop Resources Research Institute

Nigat Tilahun

Ethiopian Institute Agricultural research

\section{Desta Abebe}

Ethiopian Institute Agricultural research

\section{Worku Kebede}

Ethiopian Institute Agricultural research

\section{Research Article}

Keywords: Rice crop, Seedling growth, cold stress

Posted Date: February 10th, 2022

DOI: https://doi.org/10.21203/rs.3.rs-1323214/v1

License: (c) (i) This work is licensed under a Creative Commons Attribution 4.0 International License. Read Full License 
identification of new sources of rice genotypes (oRYZA sATIVA 1.) tolerant to cold stress

Authors: Kidist Tolosa Entele*, Richard Edema ${ }^{1}$, Jimmy Lamo $^{2}$, Nigat Tilahun ${ }^{3}$ Desta Abebe ${ }^{3}$ and Worku Kebede 3

Authors Emails: Kidist Tolosa Entele= kidisttolosa12@gmail.con, Richard Edema= redema@marcci.org, Jimmy Lamo= jlamoayo@gmail.com,Nigat Tilahun= nigatt9000@gmail.com, Desta Abebe= desta.ab12@gmail.com, Worku Kebede = workukebede121@gmail.com

${ }^{1}$ Makerere University College of agricultural science, Kampala, Uganda

${ }^{2}$ National Crop Resources Research Institute

${ }^{3}$ Ethiopian Institute Agricultural research 


\begin{abstract}
Rice crop is adversely affected by cold stress which is a common problem in the highland areas of central East Africa (Uganda, Rwanda, north east Tanzania and Kenya) and Madagascar. Cold stress is known to cause $26 \%$ to $80 \%$ yield loss. Currently, rice production in Uganda is mostly growing to the low altitude areas as there is lack of cold-tolerant varieties that can grow in the higher altitudes of the country. None of the released varieties in Uganda are tolerant cold. The few cold-tolerant lines that have been identified are short-grain types, though Ugandan consumers generally prefer long-grain size. This research aimed at identifying new sources of cold tolerant rice genotypes with preferred traits. A total of 50 lines arranged in a $5 \times 10$ alpha lattice design with two replications were used for both stressed and non-stressed experiments. Twenty one (21) days old seedlings were stressed at $10^{\circ} \mathrm{C}$ in cold air for 10 days and the other remained under normal ambient temperature. IRRI Standard Evaluation System (SES, 2013) was used to score the effect of cold stress on leaf morphology (leaf wilting score) and on leaf color (leaf yellowing score) after 7 day recovery. This study revealed eight highly cold tolerant (SCRID091-20-2-2-4, GIZA 177, NERICA 1, MET P27, MET P23, MET P60, MET P20 and MET P5) in which only GIZA 177 was short grain and fifteen tolerant varieties (MET P32, MET P11, MET P2, MET P17, MET P3, MET P24, MET P16, MET P37, MET P18, MET P9, MET P40, MET P31, MET P39, MET P22, MET P36) were identified.
\end{abstract}

Key words: Rice crop, Seedling growth, cold stress 


\subsection{Introduction}

Rice is a sensitive crop that frequently exposed to variable biotic and abiotic stress which adversely affect growth and production (GRiSP, 2013). Biotic constraints include; weeds, blast, rice yellow mottle virus (RYMV) and African rice gall midge (AfRGM). Major abiotic constraints include; extreme temperatures (heat and cold) (Dramé et al., 2013; Zhang et al., 2014), flood and drought (Odogola, 2006 ; Namazzi et al., 2010), and variable rainfall (Akongo et al., 2016) as well as soil problems (salinity, nutrient deficiencies and toxicities) (NEWEST, 2012; Dramé et al., 2013). However, cold stress is the most devastating abiotic production constraint which affects the rice plant at all growth stages from germination to grain filling, leading to high economic yield losses (Gothandam, 2012, Shinada et al., 2013). It is observed in the high altitude areas around the globe. Cold stress that occur at critical reproductive stages can adversely affect grain quality and cause yield reductions in high-altitude regions of the world (Singh et al., 2017). The loss in quality and quantity is as a result of severe reduction in seedling establishment, growth retardation, decrease tiller number, delay and incomplete grain maturation (Satake and Hayase, 1974).

In Uganda, identification of rice genotypes tolerant to cold stress is still in its exploratory stage and all the varieties under production are sensitive to the stress. Some cold tolerant rice genotypes were identified by Nyiramugisha et al. (2016) but they were short grained, sticky and non-aromatic; characteristics which is not preferred by farmers. In this study, the susceptibility and tolerance of 50 rice genotypes to cold stress was investigated. The aim was to identify rice genotypes with new sources of tolerant to cold stress and with acceptable grain size for the improvement of rice in current breeding programme.

\subsection{Materials and Methods}

\subsubsection{Genetic materials and description of study area}

Fifty (50) genotypes, including a tolerant and a susceptible check, were obtained from NaCRRI, in which their sources were from: Africa Rice (Ibadan, Nigeria), African rice (Benin), Egypt, Tanzania and Madagascar were used for the study (Table 1). The study was conducted at the Makerere University Agricultural Research Institute Kabanyolo (MUARIK) ( $0^{\circ} 28^{\prime} \mathrm{N}$ and 
$32^{\circ} 37^{\prime} \mathrm{E}$, approximately $\left.1200 \mathrm{masl}\right)$. The area receives a mean annual rainfall of $1200 \mathrm{~mm}$ with an average temperature ranging between $17^{\circ} \mathrm{C}$ and $33^{\circ} \mathrm{C}$. Screening was conducted in an artificially lighted room under controlled temperature of $10^{\circ} \mathrm{C}$, while the temperature of the screen house used to grow the control experiment was between $23^{\circ} \mathrm{C}$ and $27^{\circ} \mathrm{C}$. 
Table 1: Rice genotypes evaluated for cold tolerance at seedling stage.

\begin{tabular}{|c|c|c|c|c|c|}
\hline No. & Pedigree & Line Name & Source & Other information & Grain size \\
\hline 1 & ART35-200-2-2-B-1 & MET P10 & Africa Rice-Ibadan, Nigeria & O.barthi interspecific lines & Long \\
\hline 2 & ART27-190-1-3-3-1 & MET P27 & Africa Rice-Ibadan, Nigeria & O.barthi interspecific lines & Long \\
\hline 3 & ART35-4-1-5D-1 & MET P9 & Africa Rice-Ibadan, Nigeria & O.barthi interspecific lines & Long \\
\hline 4 & ARS126-3-B-1-2 (11) & ARS126-3-B-1-2 (11) & Africa Rice-Benin, & & Long \\
\hline 5 & ART34-79-1-2N-2 & MET P5 & Africa Rice-Ibadan, Nigeria & O.barthi interspecific lines & Long \\
\hline 6 & ART27-58-7-1-2-4-2-2 & MET P21 & Africa Rice-Ibadan, Nigeria & O.barthi interspecific lines & Long \\
\hline 7 & ART27-190-6-4-2-1-1 & MET P19 & Africa Rice-Ibadan, Nigeria & O.barthi interspecific lines & Long \\
\hline 8 & ART34-88-1-2-B-1 & MET P12 & Africa Rice-Ibadan, Nigeria & O.barthi interspecific lines & Long \\
\hline 9 & ART27-122-19-3-1-3 & MET P37 & Africa Rice-Ibadan, Nigeria & O.barthi interspecific lines & Long \\
\hline 10 & ART16-5-9-22-3-B-B-2 & MET P38 & Africa Rice-Ibadan, Nigeria & O.barthi interspecific lines & Long \\
\hline 11 & ART35-114-1-6N-2 & MET P3 & Africa Rice-Ibadan, Nigeria & O.barthi interspecific lines & Long \\
\hline 12 & ARC36-4-ET-2 (3) & ARC36-4-ET-2(3) & Africa Rice-Benin, & & Long \\
\hline 13 & ART35-52-2-7N-2 & MET P1 & Africa Rice-Ibadan, Nigeria & O.barthi interspecific lines & Long \\
\hline 14 & ART27-58-6-2-1-1-3-3 & MET P30 & Africa Rice-Ibadan, Nigeria & O.barthi interspecific lines & Long \\
\hline 15 & ART27-58-8-1-2-3 & MET P29 & Africa Rice-Ibadan, Nigeria & O.barthi interspecific lines & Long \\
\hline 16 & ART34-82-1-7N-1 & MET P2 & Africa Rice-Ibadan, Nigeria & O.barthi interspecific lines & Long \\
\hline 17 & ART27-190-7-3-2-4-3-1 & MET P32 & Africa Rice-Ibadan, Nigeria & O.barthi interspecific lines & Long \\
\hline 18 & ART27-190-6-1-4-2-2-1 & MET P26 & Africa Rice-Ibadan, Nigeria & O.barthi interspecific lines & Long \\
\hline 19 & ART34-86-2-1-B-1 & MET P11 & Africa Rice-Ibadan, Nigeria & O.barthi interspecific lines & Long \\
\hline 20 & ART35-49-1-4N-1 /MET 6 & MET P6 & Africa Rice-Ibadan, Nigeria & O.barthi interspecific lines & Long \\
\hline 21 & ART34-256-3-1-B-2 & MET P14 & Africa Rice-Ibadan, Nigeria & O.barthi interspecific lines & Long \\
\hline 22 & ARC39-145-P-2 (5) & ARC39-145-P-2 (5) & Africa Rice-Benin, & & Long \\
\hline 23 & ART27-190-1-4-2-1-1-3 & MET P40 & Africa Rice-Ibadan, Nigeria & O.barthi interspecific lines & Long \\
\hline 24 & ART27-58-7-2-2-3 & MET P20 & Africa Rice-Ibadan, Nigeria & O.barthi interspecific lines & Long \\
\hline 25 & ART27-58-6-2-2-2 & MET P28 & Africa Rice-Ibadan, Nigeria & O.barthi interspecific lines & Long \\
\hline 26 & ARC36-2-P-2-54 (2) & & Africa Rice-Benin, & & Long \\
\hline 27 & ART27-58-6-2-1-1-3-1 & MET P33 & Africa Rice-Ibadan, Nigeria & O.barthi interspecific lines & Long \\
\hline 28 & ART27-122-19-3-1-2-1-1 & MET P36 & Africa Rice-Ibadan, Nigeria & O.barthi interspecific lines & Long \\
\hline 29 & NERICA 1 & NERICA 1 & Africa Rice & Cold tolerant (Check) & Long \\
\hline 30 & ART27-58-7-1-2-2-2-2 & MET P17 & Africa Rice-Ibadan, Nigeria & O.barthi interspecific lines & Long \\
\hline
\end{tabular}




\begin{tabular}{|c|c|c|c|c|c|}
\hline 31 & ART34-76-2-8D-2 & MET P7 & Africa Rice-Ibadan, Nigeria & O.barthi interspecific lines & Long \\
\hline 32 & ART34-113-3-2-B-1 & MET P13 & Africa Rice-Ibadan, Nigeria & O.barthi interspecific lines & Long \\
\hline 33 & ART27-58-3-2-1-1 & MET P22 & Africa Rice & O.barthi interspecific lines & Long \\
\hline 34 & WITA 9 & TOX 3058-28-1-1-1 & Africa Rice & Susceptible to cold(check) & Long \\
\hline 35 & 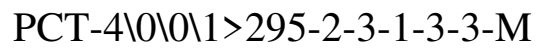 & MET P60 & Africa Rice-Ibadan, Nigeria & O.barthi interspecific lines & Long \\
\hline 36 & ART27-190-7-6-4-2 & MET P39 & Africa Rice-Ibadan, Nigeria & O.barthi interspecific lines & Long \\
\hline 37 & ARC36-2-1-2 & ARC36-2-1-2 & Africa Rice-Benin, & & Long \\
\hline 38 & ART15-7-16-38-1-B-B-2 & MET P31 & Africa Rice-Ibadan, Nigeria & O.barthi interspecific lines & Long \\
\hline 39 & ART35-100-1-7D-1 & MET P8 & Africa Rice-Ibadan, Nigeria & O.barthi interspecific lines & Long \\
\hline 40 & ART27-58-6-2-1-1-3-2 & MET P25 & Africa Rice-Ibadan, Nigeria & O.barthi interspecific lines & Long \\
\hline 41 & ART27-58-3-2-2-1 & MET P35 & Africa Rice-Ibadan, Nigeria & O.barthi interspecific lines & Long \\
\hline 42 & ART3-7L9P8-3-B-B-2-1 & MET P24 & Africa Rice-Ibadan, Nigeria & O.barthi interspecific lines & Long \\
\hline 43 & ART27-58-8-1-1-4 & MET P23 & Africa Rice-Ibadan, Nigeria & O.barthi interspecific lines & Long \\
\hline 44 & ART27-58-6-2-1-3 & MET P34 & Africa Rice-Ibadan, Nigeria & O.barthi interspecific lines & Long \\
\hline 45 & ART27-58-3-2-1-4 & MET P18 & Africa Rice-Ibadan, Nigeria & O.barthi interspecific lines & Long \\
\hline 46 & ART35-272-1-2-B-1 & MET P16 & Africa Rice-Ibadan, Nigeria & O.barthi interspecific lines & Long \\
\hline 47 & GIZA 177 & GIZA 177 & Egypt & Japonica subs pecieses & Short \\
\hline 48 & ART35-159-1-2-B-1 & MET P15 & Africa Rice-Ibadan, Nigeria & O.barthi interspecific lines & Long \\
\hline 49 & SCRID091-20-2-2-4-4 & SCRID091-20-2-2-4-4 & Tanzania & Origin Madagascar & Medium \\
\hline 50 & ART34-146-1-8N-1 & MET P4 & Africa Rice-Ibadan, Nigeria & O.barthi interspecific lines & Long \\
\hline
\end{tabular}




\subsubsection{Experimental design and data collection}

A total of fifty genotypes were tested for their reaction to cold stress following the protocol described by (Lee, 2001). Seedlings of 3-4 leaf stage (21 days after planting) were exposed to $12^{\circ} \mathrm{C} / 10^{\circ} \mathrm{C}$ (day/night) cold air temperature for 10 days in a cold growth chamber following a 12/12 hour day/night artificial lighting cycle. Ten days after stress treatment, the seedlings were taken back to normal growth conditions to allow the genotypes to recover, for seven days. The experiments were laid out in a 5 by 10 alpha lattice design, replicated twice. Two sets of experiments were established with one group exposed to cold stress while the other group was made to grow at normal temperature conditions (stress free). The seeds were planted in bin plastic pots of $24 \mathrm{~cm} \times 36 \mathrm{~cm}$ width filled with forest soil. Each genotype was planted in a pot containing twelve hills.. Di-ammonium phosphate (DAP) fertilizers (18-46-0) was applied at the rate of $0.4 \mathrm{~g} /$ pot during planting while urea (46-0-0) was top dressed at the rate of $0.4 \mathrm{~g} / \mathrm{pot}$ fifty days after germination. All agronomic management practices were done regularly as needed. Data based on visual rating (leaf growth/leaf wilting score and seedling color/leaf yellowing score) using Standard Evaluation System (SES) (IRRI, 2013), survival rate, seedling height and tiller number were collected 7 days after recovery for the stressed plants. Similar data were also collected from the non-stressed plants. However, for the non-stressed experiment, genotypes were not different for visual rating data and survival rate. As a result these parameters were not considered for further analyses. Instead, seedling height, tiller number and grain yield per pot were used for comparison. Survival rate was calculated using the following formula from (Nohrman, 1953), i.e.

Survival rate $(\%)=\frac{\text { Surviving plants }}{\text { Total number of treated plants }} * 100$

Seedling color and leaf growth were taken based on scale of 1-9 developed by IRRI (2013). The scales for these two traits were basically given by the extent of cold damage on leaf coloration and leaf morphology respectively. For seedling color, a score of 1 represents dark green seedling while a score of 9 represents dead seedling. For leaf growth, a score of 1 represents no damage on leaf and normal leaf color and hence highly tolerant while 9 represents most seedlings are dead or dying and hence highly sensitive. Therefore, based on leaf growth damage, seedling with 
scores 1-3 are considered as cold tolerant, 4-6 are intermediate and 7-9 are susceptible. The detailed description for the scales is presented in Table 2.

Table 2: Description of seedling color and leaf growth under cold stress

\begin{tabular}{|c|c|c|}
\hline Trait & Scale & Description \\
\hline \multirow{5}{*}{ Seedling color } & 1 & Dark green \\
\hline & 3 & Light Green \\
\hline & 5 & Yellowish \\
\hline & 7 & Brown \\
\hline & 9 & Dead \\
\hline \multirow{5}{*}{ Leaf growth } & $0-1$ & $\begin{array}{l}\text { No damage of leaf normal } \\
\text { leaf color (high tolerance) }\end{array}$ \\
\hline & $2-3$ & $\begin{array}{c}\text { Tip of leaves slightly dried, } \\
\text { folded (tolerant) }\end{array}$ \\
\hline & $4-5$ & $\begin{array}{c}\text { Some seedlings moderately } \\
\text { folded and wilted }\end{array}$ \\
\hline & $6-7$ & $\begin{array}{l}\text { Seedlings severely rolled } \\
\text { and dried; reddish-brown } \\
\text { leaves }\end{array}$ \\
\hline & $8-9$ & $\begin{array}{l}\text { Most seedlings dead and } \\
\text { dying or highly sensitive }\end{array}$ \\
\hline
\end{tabular}

Scaling and description according to IRRI, (2013)

\subsubsection{Data analysis}

Data were analyzed using GenStat software, $18^{\text {th }}$ edition (Payne et al., 2015) Restricted Maximum Likelihood (ReML) approach was used to generate analysis of variance (ANOVA) and to examine differences in the performance of different rice genotypes for their tolerance to cold stress. Fisher's LSD test was used to separate the means. Pearson correlation was used to examine the association among the different tolerance parameters. The model used was as follows;

$$
Y_{i j k}=\overline{\bar{Y}} \ldots+G_{i}+R_{j}+B_{k j}+E_{i j k}
$$

Where, $Y_{i j k}=$ The observed value of trait from the $\mathrm{i}^{\text {th }}$ genotype from the $\mathrm{k}^{\text {th }}$ block nested in the $\mathrm{j}^{\text {th }}$ replicate, $\overline{\overline{\mathrm{Y}}} \ldots=$ Grand mean, $\mathrm{G}_{\mathrm{i}}=$ the effect of $\mathrm{i}^{\text {th }}$ genotype, $R_{j}=$ Effect of $\mathrm{j}^{\text {th }}$ replication, 
$B_{k(j)}$ is the effect of the $\mathrm{k}^{\text {th }}$ block nested within the $\mathrm{j}^{\text {th }}$ replicate,$E_{i j k}=$ random residual or error term

Base index was estimated by the linear combination of the mean phenotypic values of the traits weighted directly by their respective economic weights (Williams 1962). Base index value was calculated as:

$I=a_{1} y_{1}+a_{2} y_{2} \ldots \ldots . a_{n} y_{n}=\sum_{i=n}^{n} a_{i} y_{i}$

Where, $y=$ Mean values, $a=$ is economic weight of the economic characters under study

Tolerance indices and reduction percentage were calculated for yield per pot for the data collected from stress and non-stress experiment. Cold tolerance indices were calculated as follow;

$S S I=(1-(Y S / Y p)) /(1-(\overline{Y S} / \overline{Y p}) \ldots .($ Fischer and Maurer, 1978)

$T O L=Y p-Y s \ldots \ldots . .($ Rosielle and Hamblin, 1981)

$Y S I=Y S / Y p^{\ldots \ldots \ldots \ldots . . . . . .(B o u s l a m a}$ and Schapaugh, 1984)

Reduction $(\%)=\left(\frac{Y p-Y s}{Y p}\right) * 100 \ldots . .($ Choukan et al., 2006)

Where $\mathrm{Ys}=$ yield under stress, $\mathrm{Yp}=$ Yield under non- stress, $\bar{Y} \mathrm{~s}=$ Yield grand mean under stress, $\bar{Y} \mathrm{p}=$ Yield grand mean under non-stress, SSI= stress susceptibility index, TOI=Tolerance index

\subsection{Results}

\subsubsection{Response of rice genotypes to cold stress}

The analysis of variance revealed a highly significant difference $(\mathrm{p}<0.001)$ among genotypes for traits collected under stress (leaf growth, seedling color, survival rate, seedling height and tiller number) (Table 3), indicating genetic variability among tested genotypes in their reaction to cold stress. However, the genotypes did not show significant difference under non-stress conditions to all the traits except seedling height significant at $\mathrm{p}<0.01$ (Table 4). 
Table 3: Mean square for rice genotypes screened at seedling stage at $10^{\circ} \mathrm{C}$

\begin{tabular}{|c|c|c|c|c|c|c|c|c|c|}
\hline Source of variation & ${ }^{1} \mathbf{D f}$ & ${ }^{2} \mathbf{L G}$ & ${ }^{3} \mathrm{SC}$ & ${ }^{4} \mathbf{S R}$ & ${ }^{5} \mathbf{S H}$ & ${ }^{6} \mathrm{TN}$ & ${ }^{7} \mathrm{GY}(\mathrm{g}) / \mathrm{pot}$ & $\%$ reduction $\mathrm{SH}$ & $\%$ reduction $\mathrm{TN}$ \\
\hline Replication & 1 & $0.01^{\mathrm{ns}}$ & $0.14 n s$ & $831.38^{* * *}$ & $2.4^{\mathrm{ns}}$ & $0.5^{* *}$ & $2197.30^{* *}$ & $768.2^{* * *}$ & $1806.2^{* * * *}$ \\
\hline Block/Replication & 8 & $0.48^{*}$ & $0.80^{* *}$ & & $6.7^{\mathrm{ns}}$ & $0.14^{\mathrm{ns}}$ & $192.50^{\mathrm{ns}}$ & $95.5^{* * *}$ & $294.4^{* * *}$ \\
\hline Genotype & 49 & $10.7^{* *}$ & $11.51^{* * *}$ & $1574.93^{* * *}$ & $97.4^{* * *}$ & $0.7^{* * *}$ & $434.31^{* *}$ & $544.9^{* * *}$ & $942.8^{* * *}$ \\
\hline Error & 41 & 0.20 & 0.26 & 63.51 & 5.6 & 0.13 & 140.90 & 53.67 & 131.3 \\
\hline${ }^{8} \mathrm{LEE}$ & $25-30$ & 0.23 & 0.36 & & 5.9 & 0.14 & 158.51 & 64.0 & 165.1 \\
\hline${ }^{9}$ SED & & 0.5 & 0.6 & 7.96 & 2.4 & 0.37 & 12.59 & 8.0 & 12.9 \\
\hline${ }^{10} \mathrm{CV}(\%)$ & & 12.05 & 14.3 & 10.7 & 9.6 & 19.8 & 24.2 & 22.9 & 35.5 \\
\hline
\end{tabular}

${ }^{*, * *, * * *}=$ significant at $0.05,0.01$ and 0.001 respectively, ns = non-significant, ${ }^{1}$ Degree of freedom ${ }^{2}$ Leaf growth, ${ }^{3}$ Seedling color, ${ }^{4}$ Survival rate,

${ }^{5}$ Seedling height, ${ }^{6}$ Tiller number, ${ }^{7}$ Grain yield in gram, ${ }^{8}$ Lattice effective error, ${ }^{9}$ Standard error of Difference, ${ }^{10}$ Coefficient of variation 
Table 4: Mean square of rice genotypes under non- stress condition

\begin{tabular}{lllll}
\hline Source of variation & ${ }^{\mathbf{1}} \mathbf{D f}$ & ${ }^{2} \mathbf{S H}$ & ${ }^{3} \mathbf{T N}$ & ${ }^{\mathbf{4}} \mathbf{G Y}(\mathbf{g}) / \mathbf{p o t}$ \\
\hline Replication & 1 & $368.1^{* * *}$ & $3.93^{\text {ns }}$ & $24.40^{\text {ns }}$ \\
Block/Replication & 8 & 16.1 & $0.96^{\text {ns }}$ & $437.40^{\text {ns }}$ \\
Genotype & 49 & $36.05^{* *}$ & $1.13^{\text {ns }}$ & $213.50^{\text {ns }}$ \\
Error & 41 & 12.37 & 0.56 & 213.70 \\
${ }^{5}$ LEE & $27-31$ & 13.42 & 0.79 & 257.28 \\
\hline${ }^{\mathbf{6}}$ SED & $\mathbf{3 . 6 6}$ & $\mathbf{0 . 8 9}$ & $\mathbf{1 6 . 0 4}$ \\
${ }^{7}$ CV $(\boldsymbol{\%})$ & $\mathbf{9 . 5 3}$ & $\mathbf{2 9 . 7 9}$ & $\mathbf{2 1 . 4}$ \\
${ }^{* * *}=$ significant 0.01, ns $=$ non-significant, ${ }^{1}$ Degree of freedom, ${ }^{2}$ Seedling height, ${ }^{3}$ Tiller number, ${ }^{4}$ Grain \\
yield in gram, ${ }^{5}$ Lattice effective Error, ${ }^{6}$ Standard error of difference, ${ }^{7}$ Coefficient of variation
\end{tabular}

\subsubsection{Performance of rice genotypes under stress and non-stress conditions}

The tested genotypes were classified into five different cold tolerance categories based on the (IRRI) (SES) scale with a little modification using leaf growth/leaf wilting score. Eight genotypes recorded score ranging from 1.0 to 1.3 were classified as highly tolerant, fifteen tolerant were classified as tolerant (score 1.7-3.1), nine were classified as moderately tolerant (score 3.2-4.8), thirteen were classified as susceptible (scores 5.5-6.6) and five classified as highly susceptible (7.6-9.0) (Table 5, Figure 1 and Appendix 1).

Table 5: Classification of rice genotypes based on their level of cold tolerance using leaf growth scale

\begin{tabular}{|l|l|c|}
\hline Category & Genotypes & Numbers \\
\cline { 2 - 3 } Very tolerant (TT) & $\begin{array}{l}\text { MET P27, GIZA 177, MET P23, SCRID091-20-2-2-4-4, } \\
\text { MET P20, NERICA 1, MET P60, MET P5 }\end{array}$ & 8 \\
\hline Tolerant (T) & $\begin{array}{l}\text { MET P32, MET P11, MET P2, MET P17, MET P3, MET } \\
\text { P24, MET P16, MET P37, MET P18, MET P9, MET P40, } \\
\text { MET P31, MET P39, MET P22, MET P36 }\end{array}$ & 15 \\
\hline $\begin{array}{l}\text { Moderate tolerant } \\
\text { MT) }\end{array}$ & $\begin{array}{l}\text { MET P34. MET P4, MET P8, MET P29, MET P35, MET } \\
\text { P15, MET P28, MET P19, MET P25 }\end{array}$ & 9 \\
\hline Susceptible (S) & $\begin{array}{l}\text { MET P1,ARC36-2-P-2-54 (2), MET P13, MET P7, MET } \\
\text { P6, MET P30,MET P12, MET P33, MET P10, MET } \\
\text { P26,MET P38, MET P14, MET P21 }\end{array}$ & 13 \\
\hline $\begin{array}{l}\text { Highly susceptible } \\
\text { (HS) }\end{array}$ & $\begin{array}{l}\text { WITA 9, ARC36-2-1-2, ARC36-4-ET-2 (3), ARC39-145- } \\
\text { P-2 (5), ARS126-3-B-1-2 (11) }\end{array}$ & 5 \\
\hline
\end{tabular}




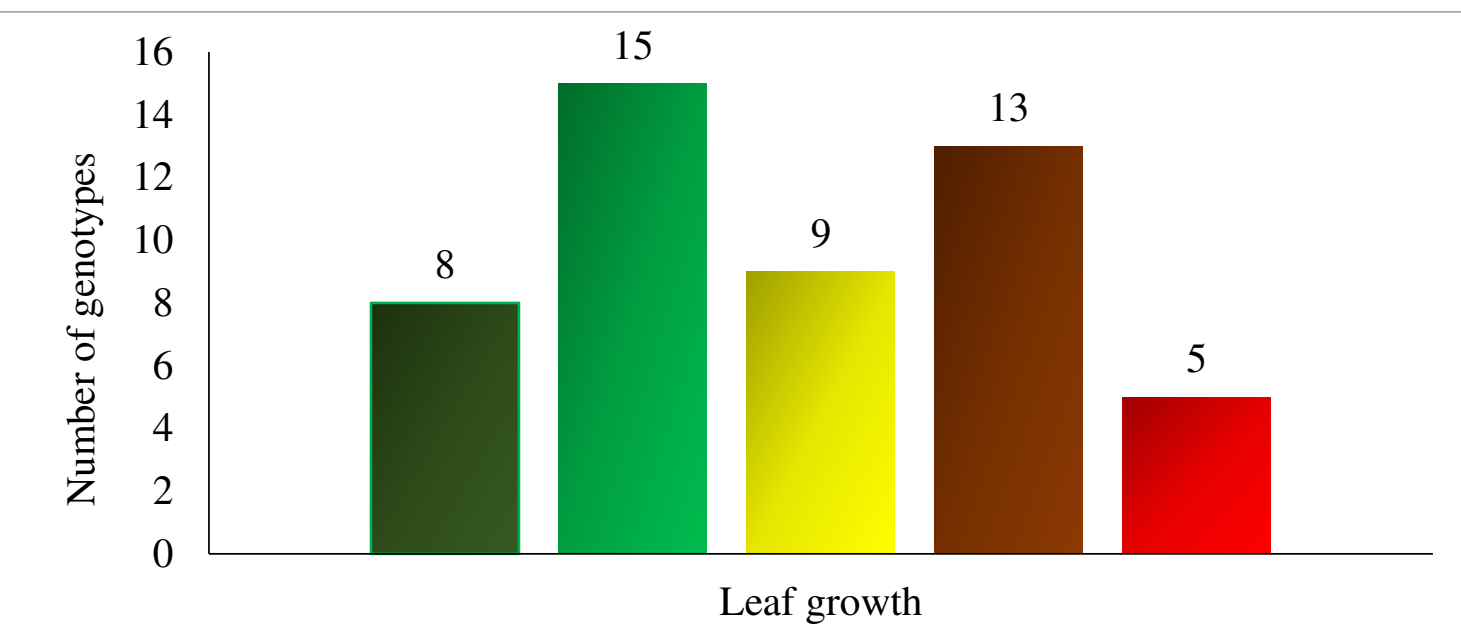

$\square$ Highly tolerant $\square$ Tolerance $\square$ Moderetly tolerant $\square$ Susceptible $\square$ Highly Susceptible

Figure 1: Reaction categories of 50 rice genotypes to cold stress at seedling stage in terms leaf wilting score after recovery

Seedling color score varied significantly $(\mathrm{p}<0.001)$ among the tested genotypes after stress treatment recovery (Table 3). The seedling of the tested genotypes fell into five color categories. Five genotypes exhibited a dark green color with the mean score ranging from 1.0 to 1.39 , eighteen were light green (mean score ranging 1.6-2.5), ten yellowish (mean score ranging 3.65.4), thirteen brownish (mean score ranging 5.6-6.9) while four seedlings dried and died (mean score ranging 8.3-9.1) (Figure 2 and Appendix 1). Genotypes ARC39-145-P-2 (5) and (ARS1263-B-1-2 (11) which were the most susceptible were completely dead after recovery. 


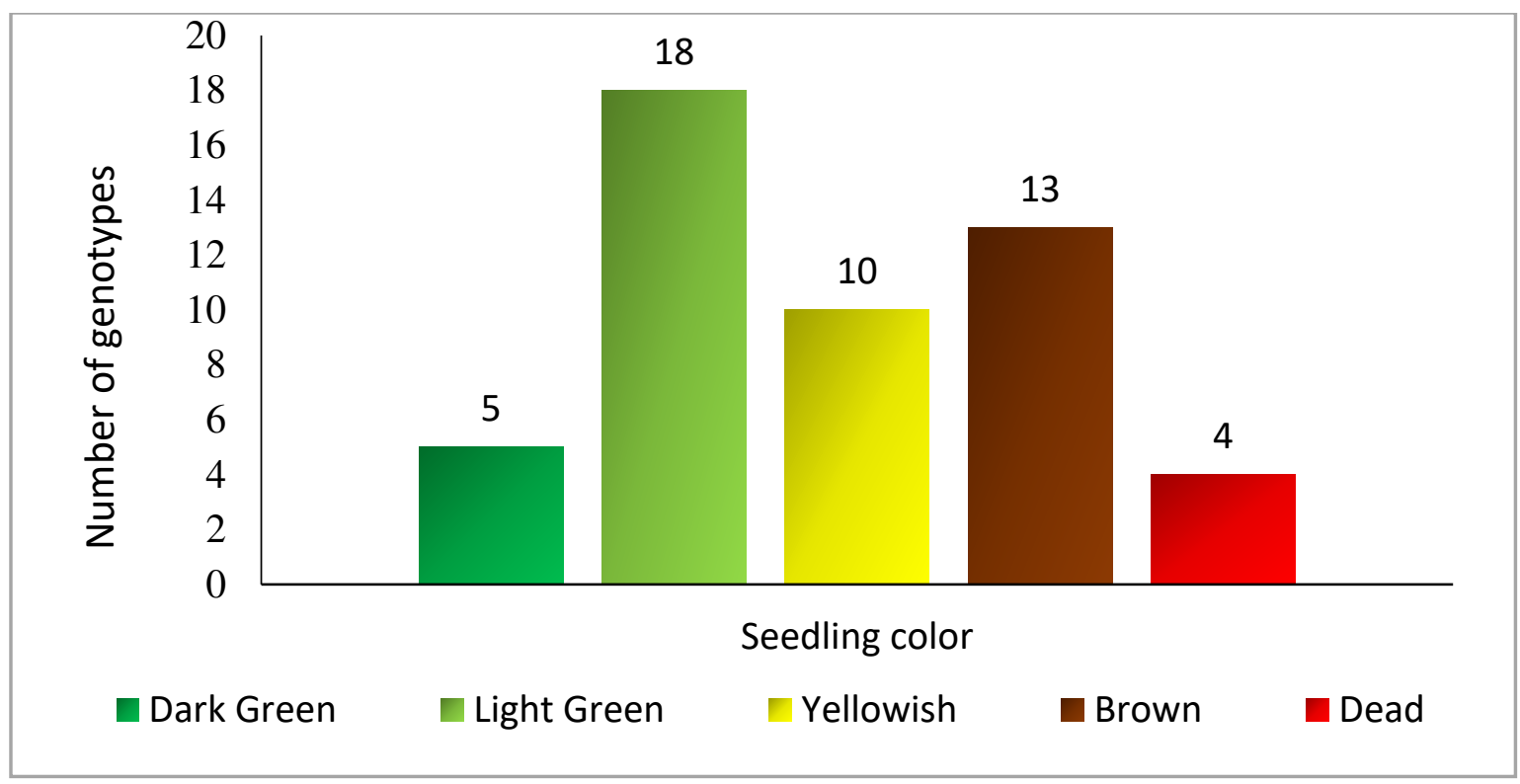

Figure 2 : Reaction categories of 50 rice genotypes to cold stress at seedling stage in terms of leaf yellowing score after recovery

Survival rate results showed a significant variation among the tested genotypes after stress recovery. It varied from $0 \%$ (completely dead) to $100 \%$ (complete seedlings survival). Highly cold tolerant genotypes exhibited $100 \%$ survival rate whereas the highly susceptible genotypes revealed low survival rate (0-33\%) (Appendix 1). The cold tolerant check (GIZA177) exhibited low score (little damage on leaf and dark green color) and high survival rate, whereas susceptible check TOX 3058-28-1-1-1/WITA 9 showed higher Score (most seedling dried and reddish brown in color) and low survival rate (Figure 3)

The evaluated genotypes varied significantly in their seedling heights both under stress and nonstress conditions (Table 3 and Table 4). Seedling height under stress ranged from nothing to 37.2 $\mathrm{cm}$ while that under non-stress conditions ranged from $24.9 \mathrm{~cm}$ to $45.1 \mathrm{~cm}$ (Appendix 1). The tallest genotype under stress was SCRID091-20-2-2-4-4 (37.2 cm) followed by MET P9 (34.1 $\mathrm{cm})$ while the shortest genotype was ARC36-2-1-2 $(8.5 \mathrm{~cm})$. Likewise, genotypes MET P9 and SCRID091-20-2-2-4-4 were the tallest under non-stress (45 cm each) (Appendix 1). In general, lower seedling heights were recorded from the stressed plants compared to the non-stressed plants (Appendix 1). Genotypes varied significantly in their reduction in seedling heights. Genotypes MET P23, MET P27, MET P 60 SCRID091-20-2-2-4-4 and NERICA 1 had the least reduction in seedling height with reduction of less than 20\%. Genotypes ARS126-3-B-1-2 (11) 
and ARC39-145-P-2 (5) were the least tolerant to cold stress with 100\% yield reduction implying that the crop was completely destroyed under cold stress (Appendix 1).

The number of tillers per hill varied significantly for genotypes evaluated under cold stress (Table 3). However, there was no significant difference among genotypes for tiller number under non- stress conditions. The number of tiller per hill ranged from 1 to 3 for stressed genotypes and 2 to 5 for non-stressed genotypes. The highest numbers of tillers were recorded for MET P60, followed by MET P27 GIZA 177, NERICA 1 and SCRID091-20-2-2-4-4, while lowest tiller number was recorded for ARC36-2-1-2 in the stressed seedlings. Reduction in tiller number for non-stress and stress condition varied significantly for the evaluated genotypes (Table 3). Genotypes MET P22, MET P60, MET P27, MET P20, MET P40, NERICA 1 and SCRID09120-2-2-4-4 had the least reduction in tiller number with reduction of less than or equal to $20 \%$. The highest reduction was recorded for genotype ARS126-3-B-1-2 (11) and ARC36-2-1-2 in which cold stress caused $100 \%$ reduction (Appendix 1).

Base index was employed to select top cold tolerant genotypes for the five main tolerance traits i.e. seedling color (SC), leaf growth (LG), survival rate (SR), tiller number (TN) and seedling height (SH). Based on the sum base index value, the best performing genotype was SCRID09120-2-2-4-4 (128.0) followed MET P27 (125.9) and MET P60 (124.8). The least performing genotype was ARS126-3-B-1-2 (11) (Table 6).

Table 6: Mean performance on main traits with base index rank of 50 rice genotypes evaluated at seedling stage in cold growth chamber

\begin{tabular}{lcccccc}
\hline Line name & ${ }^{\mathbf{1}} \mathbf{G} \mathbf{3}$ & ${ }^{\mathbf{2}} \mathbf{S C}$ & ${ }^{\mathbf{3}} \mathbf{S H}$ & ${ }^{\mathbf{4}} \mathbf{T N}$ & ${ }^{\mathbf{5}} \mathbf{S R}$ & Base index \\
\hline SCRID091-20-2-2-4-4 & 1.2 & 1.4 & 37.2 & 2.6 & 100.0 & 128.0 \\
MET P27 & 0.9 & 1.1 & 33.4 & 2.9 & 100.0 & 125.9 \\
MET P60 & 1.3 & 1.7 & 33.1 & 3.0 & 100.0 & 124.8 \\
MET 23 & 1.1 & 1.0 & 32.1 & 2.2 & 100.0 & 124.1 \\
GIZA 177 & 1.0 & 0.7 & 30.2 & 2.8 & 100.0 & 123.7 \\
MET P9 & 2.6 & 2.5 & 34.1 & 2.4 & 100.0 & 122.8 \\
MET P40 & 2.6 & 2.6 & 32.5 & 2.2 & 100.0 & 121.5 \\
MET P5 & 1.3 & 1.5 & 29.0 & 2.3 & 100.0 & 121.1 \\
NERICA 1 & 1.3 & 2.2 & 29.1 & 2.7 & 100.0 & 121.1 \\
MET P32 & 1.7 & 1.7 & 29.9 & 1.7 & 100.0 & 120.8 \\
MET P20 & 1.2 & 1.4 & 27.6 & 2.2 & 100.0 & 120.2 \\
MET P11 & 1.8 & 1.8 & 26.8 & 2.1 & 100.0 & 118.7
\end{tabular}




\begin{tabular}{|c|c|c|c|c|c|c|}
\hline MET P17 & 1.8 & 2.2 & 27.0 & 2.0 & 100.0 & 118.2 \\
\hline MET P2 & 1.8 & 1.9 & 25.6 & 2.3 & 100.0 & 117.9 \\
\hline MET P22 & 3.0 & 2.7 & 26.6 & 2.9 & 100.0 & 117.2 \\
\hline MET P24 & 2.1 & 2.2 & 26.0 & 2.0 & 100.0 & 117.2 \\
\hline MET P16 & 2.2 & 3.6 & 28.0 & 1.7 & 100.0 & 116.9 \\
\hline MET P36 & 3.1 & 1.8 & 26.1 & 2.0 & 100.0 & 116.7 \\
\hline MET P3 & 2.1 & 2.5 & 26.1 & 1.6 & 100.0 & 116.6 \\
\hline MET P37 & 2.5 & 2.7 & 29.7 & 1.9 & 95.8 & 114.8 \\
\hline MET P39 & 2.9 & 2.8 & 23.8 & 2.1 & 100.0 & 114.3 \\
\hline MET P18 & 2.5 & 3.4 & 25.2 & 2.3 & 95.9 & 111.1 \\
\hline MET P31 & 2.9 & 3.0 & 28.6 & 2.6 & 79.2 & 97.4 \\
\hline MET P4 & 3.5 & 3.9 & 23.6 & 1.5 & 79.2 & 91.0 \\
\hline MET P8 & 3.6 & 4.3 & 22.8 & 2.4 & 79.2 & 90.8 \\
\hline MET P33 & 6.2 & 4.5 & 26.2 & 1.7 & 79.2 & 89.8 \\
\hline MET P6 & 5.9 & 5.5 & 26.0 & 1.2 & 75.0 & 84.3 \\
\hline MET P35 & 3.9 & 4.1 & 25.0 & 1.4 & 70.8 & 83.0 \\
\hline MET P30 & 6.0 & 5.1 & 23.1 & 1.4 & 70.8 & 78.4 \\
\hline MET P25 & 4.8 & 3.8 & 28.4 & 2.0 & 62.5 & 77.2 \\
\hline MET P28 & 4.1 & 5.1 & 28.9 & 2.0 & 62.5 & 77.0 \\
\hline MET P15 & 4.1 & 6.1 & 27.0 & 2.2 & 62.5 & 74.7 \\
\hline MET P34 & 3.2 & 3.4 & 24.5 & 2.1 & 60.0 & 73.8 \\
\hline MET P7 & 5.8 & 5.8 & 26.4 & 1.3 & 62.5 & 72.1 \\
\hline MET P26 & 6.4 & 5.6 & 25.1 & 1.7 & 62.5 & 70.9 \\
\hline MET P14 & 6.5 & 6.5 & 25.1 & 1.9 & 62.5 & 70.3 \\
\hline MET P19 & 4.3 & 6.1 & 26.0 & 2.0 & 58.3 & 69.5 \\
\hline MET P38 & 6.5 & 5.5 & 25.8 & 1.8 & 58.3 & 67.5 \\
\hline MET P12 & 6.2 & 6.2 & 26.4 & 1.4 & 58.3 & 67.1 \\
\hline MET P21 & 6.6 & 6.7 & 25.1 & 1.3 & 58.3 & 65.3 \\
\hline MET P13 & 5.7 & 6.2 & 22.3 & 1.7 & 58.3 & 64.8 \\
\hline MET P1 & 5.5 & 5.6 & 25.1 & 1.3 & 54.2 & 63.2 \\
\hline MET P10 & 6.3 & 5.8 & 25.8 & 1.6 & 54.2 & 63.0 \\
\hline MET P29 & 3.8 & 5.9 & 25.6 & 1.8 & 50.0 & 61.3 \\
\hline WITA 9 & 7.5 & 6.9 & 17.4 & 1.8 & 33.3 & 33.7 \\
\hline ARC36-2-P-2-54 (2) & 5.7 & 6.9 & 16.9 & 1.4 & 29.2 & 30.7 \\
\hline ARC36-4-ET-2 (3) & 8.7 & 8.7 & 14.7 & 0.6 & 29.2 & 23.4 \\
\hline ARC36-2-1-2 & 7.7 & 8.3 & 8.5 & 0.5 & 12.5 & 3.4 \\
\hline ARC39-145-P-2 (5) & 9.0 & 9.1 & 0.1 & 1.0 & 0.0 & -17.0 \\
\hline ARS126-3-B-1-2 (11) & 9.0 & 9.0 & 0.1 & 0.0 & 0.0 & -17.9 \\
\hline Mean & 4.0 & 4.2 & 25.2 & 1.9 & 74.3 & \\
\hline${ }^{6} \mathrm{LSD}(5 \%)$ & 0.9 & 1.2 & 5.0 & 0.8 & 16 & \\
\hline
\end{tabular}




\subsubsection{Relationship between and among traits evaluated in the stressed experiment}

Leaf growth showed negative highly significant correlation with seedling height $\left(\mathrm{r}=-0.70^{* * * *}\right)$, survival rate $\left(\mathrm{r}=-0.87^{* * *}\right)$, tiller number $\left(\mathrm{r}=-0.75^{* * *}\right)$ and grain yield $(\mathrm{g})\left(\mathrm{r}=-0.83^{* * *}\right)$ but was positively correlated with seed color $\left(r=0.93^{* * *}\right)$. Seedling color showed negative highly significant correlation with seedling height $\left(\mathrm{r}=-0.73^{* * *}\right)$, survival rate $\left(\mathrm{r}=-0.93^{* * *}\right)$, tiller number $\left(\mathrm{r}=-0.74^{* * *}\right)$ and grain yield $\left(\mathrm{r}=-0.78^{* * *}\right)$. Seedling height, survival rate and tiller number, showed strong positive association with grain yield at $\mathrm{r}=0.66^{* * *}, 0.75^{* * *}$ and $0.67^{* * *}$, respectively (Table 7).

Table 7: Pearson correlation coefficients between morphological parameters $(n=50)$ in 50 rice genotypes exposed to cold stress of $10^{\circ} \mathrm{C}$ at seedling stage

\begin{tabular}{|c|c|c|c|c|c|}
\hline & ${ }^{1} \mathbf{L G}$ & ${ }^{2} \mathrm{SC}$ & ${ }^{3} \mathrm{SH}$ & ${ }^{4} \mathbf{S R}$ & ${ }^{5} \mathrm{TN}$ \\
\hline \multicolumn{6}{|l|}{${ }^{1} \mathrm{LG}$} \\
\hline${ }^{2} \mathrm{SC}$ & $0.93^{* * * *}$ & & & & \\
\hline${ }^{3} \mathrm{SH}$ & $-0.70^{* * * *}$ & $-0.73^{* * * *}$ & & & \\
\hline${ }^{4} \mathrm{SR}$ & $-0.87^{* * *}$ & $-0.93^{* * *}$ & $0.75^{* * *}$ & & \\
\hline${ }^{5} \mathrm{TN}$ & $-0.75^{* * * *}$ & $-0.74^{* * * *}$ & $0.70^{* * * *}$ & $0.69^{* * * *}$ & \\
\hline${ }^{6} \mathrm{GY}(\mathrm{g}) / \mathrm{pot}$ & $-0.83^{* * *}$ & $-0.78^{* * * *}$ & $0.66^{* * * *}$ & $0.75^{* * * *}$ & $0.67^{* * * *}$ \\
\hline
\end{tabular}

\subsubsection{Comparison of rice genotypes based on tolerance indices}

Performance differences between stressed and non-stressed genotypes were clearly seen after recovery and in later growth stages (Figure 3). 

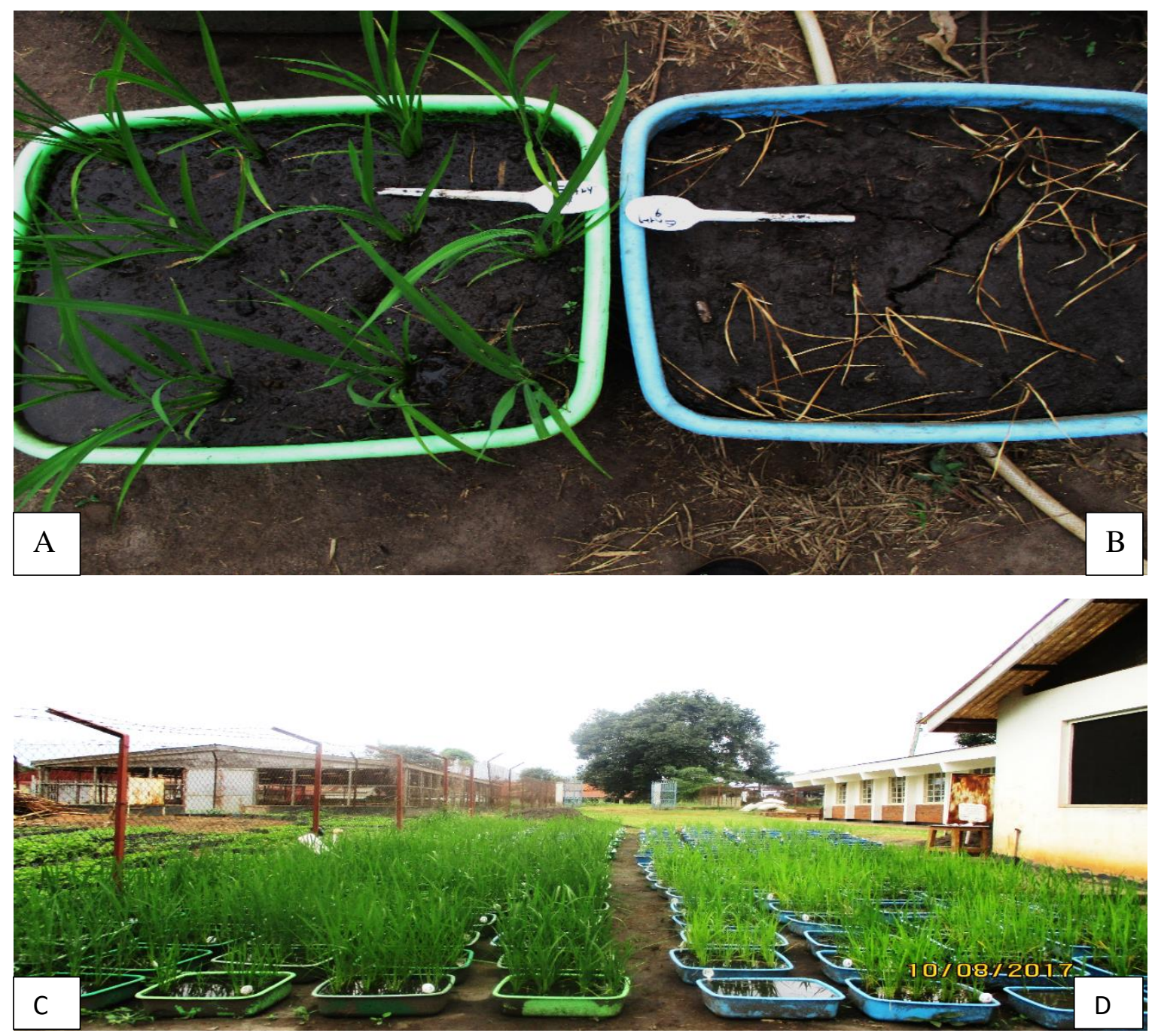

Figure 3: Effect of cold stress on rice seedlings after recovery and in later growth stage. Damage due to cold stress as observed (drying, yellowing and stunting). A and $C$ are non-stressed and $B$ and $D$ are cold stressed

Stress tolerance indices were calculated for grain yield from genotypes in the stressed and nonstressed treatments. Predicted mean of yield under stress (Ys), predicted mean of yield under non-stress (Yp), reduction percentage, stress susceptibility index (SSI), tolerance index (TOL) and yield stability index (YSI), are presented in Table 8. The reduction rate ranged from 7.33 to $100 \%$ for grain yield. The lowest reduction in grain yield was recorded for genotype SCRID09120-2-2-4-4 (7.33\%) followed by MET P20 (7.97\%), indicating tolerance to cold stress. Highest yield reduction percentage was recorded for ARC39-145-P-2 (5) and ARS126-3-B-1-2 (11) at $100 \%$, suggesting high susceptibility to cold stress. The SSI value ranged from 0.24 to 3.29 . The lower the SSI value the more tolerant the genotypes is. The lowest SSI value, was calculated for 
genotype SCRID091-20-2-2-4-4 (0.24), followed by MET P20 (0.26) and MET P27 (0.27) indicating that these genotypes were cold tolerant. On the other hand, a high SSI value (3.29) was calculated for ARS126-3-B-1-2 (11) and ARC39-145-P-2 (5) suggesting that these two genotypes are the most susceptible to cold stress. TOL value ranged from 4.35 to $76.26 \mathrm{~g}$. The lower TOL value (yield difference) was observed for genotype MET P27 (4.35g) followed by MET P20 (5.47g) and SCRID091-20-2-2-4-4 (5.68g). The Highest yield differences between stress and non-stress was observed for genotype ARC39-145-P-2 (5) (76.26g), suggesting susceptibility to cold stress. YSI, which is the ratio of yield under stress to non-stress ranged from 0 (ARS126-3-B-1-2 (11) and ARC39-145-P-2 (5)) to 0.93 (SCRID091-20-2-2-4-4) (Table 8 and Appendix 2).

Table 8: Tolerance indices of the best 23 cold tolerance and four highly susceptible rice genotypes under stress and non-stress conditions for grain yield

\begin{tabular}{lllllll}
\hline Line name & $\begin{array}{l}\text { GY }(\mathbf{g}) / \mathbf{p o t} \\
(\mathbf{s t r e s s e d})\end{array}$ & $\begin{array}{l}\mathbf{G Y}(\mathbf{g}) / \mathbf{p o t} \\
(\text { Non-stressed) }\end{array}$ & $\begin{array}{l}\text { Reduction } \\
(\boldsymbol{\%})\end{array}$ & SSI & TOI & YSI \\
\hline SCRID091-20-2-2-4-4 & 71.8 & 77.5 & 7.3 & 0.2 & 5.7 & 0.9 \\
MET P20 & 63.1 & 68.6 & 8.0 & 0.3 & 5.5 & 0.9 \\
MET P27 & 49.4 & 53.7 & 8.1 & 0.3 & 4.4 & 0.9 \\
MET P5 & 73.0 & 79.6 & 8.2 & 0.3 & 6.5 & 0.9 \\
GIZA 177 & 64.8 & 72.0 & 9.9 & 0.3 & 7.2 & 0.9 \\
MET P60 & 61.8 & 69.4 & 11.0 & 0.4 & 7.6 & 0.9 \\
NERICA 1 & 85.9 & 97.1 & 11.5 & 0.4 & 11.2 & 0.9 \\
MET P40 & 49.7 & 56.4 & 12.0 & 0.4 & 6.7 & 0.9 \\
MET P23 & 69.0 & 79.0 & 12.7 & 0.4 & 10.0 & 0.9 \\
MET P3 & 57.6 & 66.5 & 13.3 & 0.4 & 8.8 & 0.9 \\
MET P18 & 76.3 & 88.1 & 13.4 & 0.4 & 11.8 & 0.9 \\
MET P2 & 63.4 & 74.2 & 14.5 & 0.5 & 10.8 & 0.9 \\
MET P32 & 63.7 & 74.9 & 15.0 & 0.5 & 11.2 & 0.9 \\
MET P37 & 53.9 & 64.1 & 15.9 & 0.5 & 10.2 & 0.8 \\
MET P11 & 72.9 & 88.6 & 17.7 & 0.6 & 15.7 & 0.8 \\
MET P17 & 65.0 & 79.1 & 17.8 & 0.6 & 14.1 & 0.8 \\
MET P9 & 66.3 & 81.4 & 18.5 & 0.6 & 15.1 & 0.8 \\
MET P16 & 49.8 & 61.6 & 19.2 & 0.6 & 11.9 & 0.8 \\
MET P36 & 68.4 & 85.6 & 20.1 & 0.7 & 17.2 & 0.8 \\
MET P39 & 46.1 & 57.8 & 20.3 & 0.7 & 11.7 & 0.8 \\
MET P31 & 61.5 & 77.6 & 20.7 & 0.7 & 16.1 & 0.8 \\
MET P22 & 54.9 & 69.7 & 21.2 & 0.7 & 14.8 & 0.8 \\
MET P24 & 56.6 & 73.5 & 22.9 & 0.8 & 16.9 & 0.8
\end{tabular}




\begin{tabular}{lllllll} 
ARC36-2-1-2 & 30.2 & 83.0 & 63.7 & 2.1 & 52.8 & 0.4 \\
ARC36-4-ET-2 (3) & 11.6 & 63.1 & 81.7 & 2.7 & 51.6 & 0.2 \\
ARS126-3-B-1-2 (11) & 0.0 & 71.4 & 100.0 & 3.3 & 71.4 & 0.0 \\
ARC39-145-P-2 (5) & 0 & 76.3 & 100.0 & 3.3 & 76.3 & 0.0 \\
\hline Mean & 52.1 & 74.8 & & & \\
\hline LSD (5\%) & 29.1 & Ns & & & \\
\hline GY (g) = (Grain Yield in gram), TOL=Tolerance index, SSI= Stress Susceptibility Index, YSI= Yield
\end{tabular}

Susceptibility Index LSD= Least Significant Difference and ns=non-significant 


\subsection{Discussion}

The study demonstrates the existence of new sources of tolerance to cold stress in the new rice genotypes which could be used to introgress cold tolerance into farmers' preferred but cold susceptible rice cultivars. Substantial variations were observed among the tested rice genotypes for cold stress parameters such as leaf growth/leaf wilting and seedling color/leaf yellowing, survival rate, tiller number and seedling height (Table 5).

Leaf growth/leaf wilting score was able to distinguish the tolerant and susceptible genotypes. Similarly, Andaya and Mackill (2003), were able to identify highly cold tolerant rice genotypes at seedling stage using leaf grow/leaf wilting score and suggested that leaf growth could be the most suitable parameter in evaluating cold tolerance at seedling stage. The evaluated genotypes were grouped into five categories based on their reaction to cold treatments. The categories include; highly tolerant (8 genotypes), tolerant (15 genotypes), moderately tolerant (9 genotypes), susceptible (13 genotypes) and highly susceptible (5 genotypes). Of the 8 highly tolerant genotypes 5 genotypes i.e. MET P27, MET P23, MET P20, MET P60 and MET P5 were newly identified while the other 3 genotypes i.e. GIZA177, SCRID091-20-2-2-4-4 and NERICA 1 had previously been reported show tolerance to cold stress. SCRID091-20-2-2-4-4 by Nyiramugisha et al. (2017), GIZA 177 by Suh et al. (2013) and NERICA 1 by Dessie et al. (2014) and Wainaina et al. (2015). In addition, GIZA 177 is a Japonica subspecies. Japonica subspecies are known to be cold tolerant as confirmed by Cruz and Milach (2004) and Park et al. (2013). SCRID091-20-2-2-4-4 has background of origin of Madagascar, which is one of the places were rice is grown mostly in the highland parts of the country. The newly identified

highly cold tolerant genotypes were from African rice Ibadan Nigeria and they were from the O.barthi interspecific line. Even though, GIZA 177 is a highly cold tolerant genotype, it is of a short grain type which is not preferred by Ugandan farmer. However, it can be a good source of cold tolerance for breeding programme improving farmer preferred varieties. SCRID091-20-2-24-4 is a medium grain size variety. The other newly identified genotypes (i.e. MET P27, MET P23, MET P60, MET P20 and MET P5 have long grain sizes preferred by Ugandan farmers. Leaf growth/leaf wilting scores were significantly $(\mathrm{p}<0.001)$ and negatively correlated survival rate, tiller number, seedling height and grain yield (g) per pot (Table 9), suggesting the lower the score the better performance of genotypes. There was strong positive correlation between leaf 
growth and seeding color scores $\left(\mathrm{r}=0.93^{* * *}\right)$ suggesting that selection can be done using any of the traits.

The loss of chlorophyll content observed in rice seedlings was evaluated using seedling color/leaf yellowing scores. The tested genotypes were grouped into five different leaf color categories based on seeding color observed after recovery. The categories included dark green (with 5 genotypes), light green (with 18 genotypes), yellowish (with 10 genotypes), brownish (with 13 genotypes) and mostly dried and dead seedlings (with 4 genotypes). Cold stress heavily affects chlorophyll content, mainly exhibited though leaf discoloration which finally weakens the photosynthetic ability of the seedling (Lou et al., 2007; Kim et al., 2012; Pradhan and Rani, 2017). Studies conducted by Kim et al. (2012) and Donoso et al. (2015) confirmed that seedling color scores was related to chlorophyll content. In this study, seeding color was significantly and negatively correlated with survival rate, seedling height, tiller number and finally grain yield. This suggested that seedlings with green color (i.e lower score) had good survival, height, tiller and grain yield. In other words, genotypes with lower seedling color scores had better photosynthetic ability than seedlings with higher scores. Similarly, Ranawake and Nakamura (2011) reported that genotypes with green color exhibited high height in their evaluation of inbred lines for cold tolerance at seedling stage.

Assessment of survival rate was effective in identifying degree of recovery in genotypes. Cold tolerant genotypes recovered up to $100 \%$, whereas susceptible genotypes were unable to recover and/or had low recovery from the applied cold stress. These results were similar to Kim et al. (2012) in their study conducted to evaluate rice seedlings tolerance to constant and intermittent low temperature stress observed good recovery. Survival rate was significantly and positively correlated with seedling height, tiller number and grain yield per pot. The higher survival rate the more tolerant the genotypes and the better the performance in later growth.

Seedlings were stunted under applied cold treatment. This was clearly observed by comparing seedlings under stress and non-stressed conditions. Seedling height under stress ranged from 8.51 to $37.2 \mathrm{~cm}$ and 24.9 to $45.2 \mathrm{~cm}$ for those in non-stress conditions. Stunting at seedling stage is of the major indicators of cold stress, as reported by Yoshida (1981); Pradhan et al. (2017) and Zhang et al. (2014). In this study, seedling height was positively correlated with survival rate, tiller number and grain yield per pot. 
Examination of tillering is one useful way to examine the growth status of rice plants (Yoshida, 1981). In this study, tiller number per hill was ranged from 1 to 3 and 2 to 5 for the stressed and non-stressed experiment respectively. Lower tiller number was exhibited under stressed conditions suggesting that cold stress affected tillering ability. Similar result were obtained Shimono et al. (2007) and Ndour et al. (2016) who reported that low temperature at seedling stage reduced tillering ability.

Twenty three best cold tolerant rice genotypes were selected by base index selection using five traits, for consistent performance under highland parts of Uganda. Base index is a method of selection where indices are established by the linear combination of the mean phenotypic values of the characters weighted directly by their respective economic weights (Williams, 1962). Economic weight was given to the traits based on their importance to identify cold tolerance. As Biosci et al. (2013) reported economic weight is given to certain traits based on breeding objectives and their importance or contribution in selecting genotypes of interest. Traits with high genetic variance had the power to distinguish tolerance and susceptibility therefore received high economic weights (i.e LG, SC, SR and TN). Traits with low genetic variance had less power in distinguishing the cold tolerance and susceptible genotypes and received low economic weight (i.e SH). Based on the index (the sum value of the five traits), SCRID091-20-2-2-4-4 was ranked first followed by MET P27. The lowest value was obtained for ARS126-3-B-1-2 (11). The highest index value for SCRID091-20-2-2-4-4 showed that this genotype performed relatively better for the five collected traits and revealed its tolerance to cold stress. The lower index value of ARS126-3-B-1-2 (11) genotype pointed to the susceptibility of the genotype to cold stress. Base index selection was able to identify and group the cold tolerant and susceptible rice genotypes in this screening condition which fitted well with visual selection and tolerance indices selection.

Tolerance indices tell us the amount of yield reduction due to cold stress as compared to nonstress conditions. The cold stress treatment used in this study caused up to $100 \%$ yield reduction for highly susceptible genotypes. Low Stress Susceptibility Index (SSI) value implied tolerance of the genotypes under applied stress. SCRID091-20-2-2-4-4 followed by MET P20 had low SSI value. Numerous studies confirmed that the lower the SSI value, the more tolerant the genotype is and it was reported as a good index to identify stress tolerance and susceptible genotypes 
(Guttieri et al., 2001; Talebi et al (2009) ; Shiranirad and Abbasian, 2011; Zdravković et al., 2013). High Tolerance index (TOL) value indicated high loss due to the applied stress and susceptibility of the genotypes. The lower the TOL value, the more desirable the genotypes (Rosielle and Hamblin, 1981). Based on this approach the desired genotypes in this study was SCRID091-20-2-2-4-4 which had low TOL value, followed by MET P20 and the undesirable genotype was ARC39-145-P-2 (5). The higher yield under stress to non-stress ration (YSI) suggested tolerance of genotypes (Bouslama and Schapaugh, 1984). In all values of tolerance indices, SCRID091-20-2-2-4-4 ranked first followed by MET P20 while the worst genotype was ARS126-3-B-1-2 (11) which ranked last for tolerance indices. According to this study, yield reduction rate, TOL, SSI and YSI were the best fitted tolerance indices to identify cold tolerant genotypes. A similar study reported that these indices were the more powerful stress discriminators in severe cold stress condition (Talebi et al., 2009). In general, tolerance indices were able to distinguish genotypes which tolerate cold stress and showed small performance difference as compared to non-stressed treatment. This revealed that genotypes with low TOL, SSI and YSI can give better yield in presence of cold stress. In other words, farmers may face lower yield penalty if they grew these varieties with low TOL, SSI, and YSI.

\subsection{Conclusions and Recommendation}

This study identified 8 highly cold tolerant, 15 (tolerance), 9 (moderately tolerant), 13 (susceptible) and 5 (highly susceptible) rice genotypes from 50 rice genotypes tested at seedling stage. MET P27, GIZA 177, MET P23, SCRID091-20-2-2-4-4, NERICA 1, MET P5, MET P20 and MET P60 were the highly cold tolerant rice genotypes selected for all applied selection methods. Apart from these (GIZA 177, SCRID091-20-2-2-4-4 and NERICA 1), the other identified cold tolerant genotypes had long grain size. GIZA 177 (short grain), SCRID091-20-22-4-4 (medium grain size) and NERICA 1 were also identified as cold tolerance in the other study. These short and medium grain size rice could be used as donors in breeding for cold tolerance.

\section{Abbreviations}

Df. Degree of freedom

GRiSP Global Rice Science Partnership 
IRRI International Rice Research Institute

LSD Least Significance Difference

MUARIK Makerere University Agricultural Research Institute Kabanyolo

NaCRRI National Crop Resources Research Institute

NEWEST Nitrogen- use Efficient, Water-use Efficient and Salt Tolerance

ReML Restricted Maximum Likelihood

SES Standard Evaluation System

SSI Stress Susceptibility Index

STI Stress Tolerance Index

TOL Tolerance Index

YP Yield under non-stress

YS Yield under stress

\section{Declaration}

\section{Ethical approval and consent to participate}

No applicable

\section{Consent for publication}

No applicable

\section{Availability of supporting data}

All data generated and analyzed in this study included in the figures and tables presented in the manuscript

\section{Competing interest:}

No competing interest

\section{Funding}

This work was funded by scholarship from Alliance for a Green Revolution in Africa (AGRA) Authors' contributions 
Kidist Tolosa was designed research and performed research and wrote the manuscript,Richard Edema, Jimmy Lamo supervised research, Nigat Tilahun, Desta Abebe and Worku kebede help in analyzed and edited the manuscript. The author(s) read and approved the final manuscript.

\section{Author details}

Department of Tef crop Improvement program, Debre Zeit research center, P.O.Box 32 Bishoftu Ethiopia under Ethiopian Institute Agricultural research, P.O.Box 2003. v

Corresponding author details: Crop breeder, Email: kidisttolosa12@gmail.com

\section{Acknowledgments}

This research was carried out with the support of Alliance for a Green Revolution in Africa (AGRA) project. I'm very grateful for the awarded scholarship. Many thanks to Dr. Richard Edema for effective coordination, administration. The commendable guidance, advice and positive criticism from my supervisors; Dr. Richard Edema and Dr. Jimmy Lamo were the backbone for the success of this work and words cannot express my thanks to them.

My heartfelt thanks also go to Professor Paul Gibson for building me with the subject matter. I feel elated in expressing thanks to my host institution Makerere University and institutions that helped me to conduct the research experiments. The National Crop Resources Research Institute (NaCRRI) for giving me research material and many thanks to for the technician in these institution especially, Fredrick Ocuna from NaCRRI. A lot thanks to Issa Obed who helped me during screen work. I'm very grateful to Ethiopian Institute of Agricultural Research (EIAR) for giving me study leave and Debre Zeit research center for all support and encouragement you have given me.

\section{Reference}

Andaya, V.C., Mackill, D.J., 2003. Mapping of QTLs associated with cold tolerance during the vegetative stage in rice. Journal of Experimental Botany. 54, 2579-2585. doi:10.1093/jxb/erg243.

Biosci, I.J., Safari, P., Honarnejad, R., Esfahani, M., 2013. Indirect selection for increased oil yield in peanut: Comparison selection indices and biplot analysis for simultaneous improvement multiple traits. Int. J. Biosci. 3, 87-96. 
Bouslama, M., Schapaugh, W.T., 1984. Stress tolerance in soybeans. I. evaluation of three screening techniques for heat and drought tolerance. Crop Sci. 24, 933. doi:10.2135/cropsci1984.0011183X002400050026x.

Choukan, R., Taherkhani, T., Ghannadha, M.R., Khodarahmi, M., 2006. Evaluation of drought tolerance in grain maize inbred lines using drought tolerance indices. Iran. J. Agric. Sci. 8, 79-89.

Cruz, R.P., Duarte, I.T.L., Cabreira, C., 2010. Inheritance of rice cold tolerance at the seedling stage. Sci. Agric. 67, 669-674. doi:10.1590/S0103-90162010000600008.

Cruz, R.P., Milach, S.C.K., 2004. Cold Tolerance at the Germination stage of Rice : Methods of Evaluation and Characterization of Genotypes. Sci. Agric. 1-8.

Dessie, A., Kenji, I., Shiwachi, H., 2014. Phenotypic expression in upland NERICA rice under low temperature condition at germination stage. Int. J. Agric. Sci. Nat. Resour. 1, 107-114.

Donoso, G., Cabas, P., Paredes, M., Becerra, V., Balzarini, M., 2015. Cold tolerance evaluation of temperate rice ( Oryza sativa L . ssp . japonica) genotypes at seedling stage. Gayana Bot. 72, 1-13.

Falconer, D.S., 1996. Introduction to quantitative genetics, Fourth edition. Longman : Essex.

Fischer, Maurer, R., 1978. Drought resistance in spring wheat cultivars . I. Grain yield response. Aust.J. Agric.Res 29, 897-912.

Guttieri, M.J., Stark, J.C., Brien, K.O., Souza, E., 2001. Relative sensitivity of spring wheat grain yield and quality parameters to moisture deficit. Crop Sci. 41, 327-335. doi:10.2135/cropsci2001.412327x.

IRRI, 2013. Standard Evaluation System ( SES ) for rice, fifth Edition. International rice research institute, Manila, Philippines.

Kim, S.I., Kim, D.M., Tai, T.H., 2012. Evaluation of rice seedling tolerance to constant and intermittent low temperature stress. Rice science 19, 295-308. doi:10.1016/S16726308(12)60054-7.

Lee, M., 2001. Low temperature tolerance in rice: The Korean experience, in: Shu Fukai, Basnayake, J. (Eds.), Increased lowland rice production in the Mekong region ACIAR Proceedings 101. IRRI, Makati, Philippines. 
Ndour, D., Diouf, D., Bimpong, I., Sow, A., Kanfany, G., Manneh, B., 2016. Agromorphological evaluation of rice (Oryza sativa L.) for seasonal adaptation in the sahelian environment. Agronomy 6, 8. doi:10.3390/agronomy6010008

Nohrman, B. A., 1953. Survival rate calculation. Acta radiol. 39, 78-82. doi:10.3109/00016925309136688

Nyiramugisha, J., Lamo, J., Oloka, B.M., Ongom, P., Gibson, P., Edema, R., 2017. Response to cold stress at reproductive stage of introduced and adapted rice genotypes in Uganda. RUFORUM Work. Doc. Ser. (ISSN 1607-9345) No. 14633 - 638. Available from http//repository.ruforum.org 14, 633-638.

Park, I.K., Oh, C.S., Kim, D.M., Sang, M.Y., Sang, N.A., 2013. QTL mapping for cold tolerance at the seedling stage using introgression lines derived from an intersubspecific cross in rice. Plant Breed. Biotech 1, 1-8. doi:10.9787/PBB.2013.1.1.001Payne, R., Murray, D., Harding, S., 2015. An introduction to the Genstat command language (18th Edition), 18th editi. ed. VSN International.

Pradhan, S., Rani, K.J., 2017. Screening techniques to measure cold tolerance in rice. Journal of Pharmacognosy and Phytochemistry 6, 781-785.

Pradhan, S., Rani, K.J., Raju, Ch., 2017. Cold tolerance in rice at seedling and reproductive stage. Pharmacogn. Phytochem. 6, 984-988.

Ranawake, A., Nakamura, C., 2011. Cold tolerance of an inbred line population of rice (Oryza sativa L.) at different growth stages. Tropical Agricultural Research \& Extension 14.

Rosielle, A.A., Hamblin, J., 1981. Theoretical aspects of selection for yield in stress and nonStress environments. Crop Sci. 21, 943-946.

Satake, T., Hayase, H., 1974. Male sterility caused by cold treatment at young microspore stage in rice plant, in: Proceedings of the crop science society of Japan. pp. 36-39.

Shimono, H., Okada, M., Kanda, E., Arakawa, I., 2007. Low temperature-induced sterility in rice: Evidence for the effects of temperature before panicle initiation. Field Crops Research 101, 221-231. doi:10.1016/j.fcr.2006.11.010Shinada, H., Iwata, N., Sato, T., Fujino, K., 2013. 
Genetical and morphological characterization of cold tolerance at fertilization stage in rice. Breed. Sci. 63, 197-204. doi:10.1270/jsbbs.63.197

Shiranirad, A., Abbasian, A., 2011. Evaluation of drought tolerance in rapeseed genotypes under non stress and drought stress conditions. Not. Bot. Horti . Agrobo. 39, 164-171.

Singh, B.K., Sutradhar, M., Singh, A.K., Mandal, N., 2017. Cold stress in rice at early growth stage: An overview. Int. J. Pure App. Biosci. 5(2) 407-419 5, 407-419. doi:http://dx.doi.org/10.18782/2320-7051.2750Sivasubramanian, S., Menon, M., 1973. Heterosis and inbreeding depression in rice. Madras Agric. J. 60.

Suh, J., Cho, Y., Lee, J., Lee, S., Jung, J., Choi, I., Kim, M., Kim, C., Jena, K.K., 2013. SSR analysis of genetic diversity and cold colerance in temperate rice germplasm. Plant Breed. Biotech. 1, 103-110. doi:http://dx.doi.org/10.9787/PBB.2013.1.2.103Talebi, R., Fayaz, F., Naji, A.M., 2009. Effective selection criteria for assessing drought stress tolerance in durum wheat (Triticum durum Desf.). Gen. Appl. Plant Physiol. 35, 64-74.

Wainaina, C.M., Inukai, Y., Masinde, P.W., Ateka, E.M., Murage, H., Kano-Nakata, M., Nakajima, Y., Terashima, T., Mizukami, Y., Nakamura, M., Nonoyama, T., Saka, N., Asanuma, S., Yamauchi, A., Kitano, H., Kimani, J., Makihara, D., 2015. Evaluation of cold tolerance in NERICAs compared with Japanese standard rice varieties at the reproductive stage. J. Agron. Crop Sci. 201, 461-472. doi:10.1111/jac.12125

Williams, J.S., 1962. The Evaluation of a selection index. Int. Biometric Soc. 18, 375-393.

Yoshida, S., 1981. Fundamentals of rice crop science. IRRI, Los Baños (Philippines).

Zdravković, J., Jovanović, Z., Djordjević, M., Girek, Z., Zdravković, M., Stikić, R., 2013. Application of stress susceptibility index for drought tolerance screening of tomato populations. Genetika 45, 679-689. doi:10.2298/GENSR1303679Z

Zhang, Q., Chen, Q., Wang, S., Hong, Y., Wang, Z., 2014. Rice and cold stress: Methods for its evaluation and summary of cold tolerance-related quantitative trait loci. Springer open Journal 7, 24. doi:10.1186/s12284-014-0024-3 
APPENDICES

Appendix 1: Means performance of 50 rice genotypes screened at seedling stage (at $10{ }^{\circ} \mathrm{C}$ for 10 days) and their comparison mean in non-stress condition

\begin{tabular}{|c|c|c|c|c|c|c|c|c|c|c|c|}
\hline \multirow[t]{2}{*}{ Line name } & \multirow{2}{*}{$\begin{array}{c}\begin{array}{c}\text { Leaf } \\
\text { growth }\end{array} \\
\text { Stressed }\end{array}$} & \multirow{2}{*}{$\begin{array}{l}\begin{array}{l}\text { Seedling } \\
\text { Color }\end{array} \\
\text { Stressed }\end{array}$} & \multicolumn{2}{|c|}{$\begin{array}{l}\text { Seedling } \\
\text { height }(\mathrm{cm})\end{array}$} & \multirow[t]{2}{*}{$\begin{array}{c}\text { Reduction } \\
\text { rate }(\%)\end{array}$} & \multicolumn{2}{|c|}{ Tiller number } & \multirow[t]{2}{*}{$\begin{array}{c}\text { Reduction } \\
\text { rate }(\%)\end{array}$} & \multirow{2}{*}{$\begin{array}{l}\text { Survival } \\
\text { rate }\end{array}$} & \multicolumn{2}{|c|}{$\begin{array}{l}\text { Grain yield }(\mathrm{g}) / \\
\text { pot }\end{array}$} \\
\hline & & & Stressed & N-stressed & & Stressed & N-stressed & & & Stressed & N-stressed \\
\hline MET 10 & 6.3 & 5.8 & 25.8 & 44.8 & 42.4 & 1.6 & 3.2 & 49.6 & 54.2 & 32.8 & 82.6 \\
\hline MET 27 & 1.0 & 1.1 & 33.4 & 38.9 & 14.2 & 2.9 & 3.3 & 12.7 & 100.0 & 49.4 & 53.7 \\
\hline MET 9 & 2.6 & 2.5 & 34.1 & 45.1 & 24.6 & 2.4 & 3.0 & 20.7 & 100.0 & 66.3 & 81.4 \\
\hline ARS126-3-B-1-2 (11) & 9.0 & 9.0 & 0.1 & 30.9 & 99.8 & 0.0 & 3.7 & 100.1 & 0.0 & 0.0 & 71.4 \\
\hline MET 5 & 1.3 & 1.5 & 29.0 & 38.1 & 23.9 & 2.3 & 3.1 & 27.1 & 100.0 & 73.0 & 79.6 \\
\hline MET 21 & 6.6 & 6.7 & 25.1 & 37.6 & 33.1 & 1.3 & 2.3 & 41.3 & 58.3 & 45.8 & 85.7 \\
\hline MET 19 & 4.3 & 6.1 & 26.0 & 38.4 & 32.3 & 2.0 & 2.7 & 26.4 & 58.3 & 51.8 & 74.7 \\
\hline MET 12 & 6.2 & 6.2 & 26.4 & 39.9 & 33.8 & 1.4 & 3.3 & 57.4 & 58.3 & 49.4 & 90.1 \\
\hline MET 37 & 2.5 & 2.7 & 29.7 & 42.7 & 30.5 & 1.9 & 3.0 & 36.4 & 95.8 & 53.9 & 64.1 \\
\hline MET 38 & 6.5 & 5.5 & 25.8 & 40.9 & 37.1 & 1.8 & 3.0 & 42.0 & 58.3 & 34.5 & 83.4 \\
\hline MET3 & 2.1 & 2.5 & 26.1 & 37.3 & 30.0 & 1.6 & 2.2 & 27.6 & 100.0 & 57.6 & 66.5 \\
\hline ARC36-4-ET-2 (3) & 8.7 & 8.7 & 14.7 & 32.0 & 54.2 & 0.6 & 3.7 & 83.8 & 29.2 & 11.6 & 63.1 \\
\hline MET 1 & 5.5 & 5.6 & 25.1 & 39.0 & 35.8 & 1.3 & 2.6 & 50.4 & 54.2 & 43.2 & 73.3 \\
\hline MET 30 & 6.0 & 5.1 & 23.1 & 32.4 & 28.6 & 1.4 & 3.1 & 56.1 & 70.8 & 38.6 & 68.3 \\
\hline MET29 & 3.8 & 5.9 & 25.6 & 39.3 & 35.0 & 1.8 & 2.5 & 26.5 & 50.0 & 57.4 & 80.9 \\
\hline MET 2 & 1.8 & 1.9 & 25.6 & 38.8 & 34.1 & 2.3 & 2.9 & 18.1 & 100.0 & 63.4 & 74.2 \\
\hline MET 32 & 1.7 & 1.7 & 29.9 & 43.4 & 31.1 & 1.7 & 2.6 & 32.6 & 100.0 & 63.7 & 74.9 \\
\hline MET 26 & 6.4 & 5.6 & 25.1 & 40.2 & 37.6 & 1.7 & 2.8 & 41.4 & 62.5 & 50.8 & 86.7 \\
\hline MET 11 & 1.8 & 1.8 & 26.8 & 41.4 & 35.2 & 2.1 & 3.1 & 32.2 & 100.0 & 72.9 & 88.6 \\
\hline MET 6 & 5.9 & 5.5 & 26.0 & 39.7 & 34.5 & 1.2 & 2.4 & 50.3 & 75.0 & 44.3 & 67.5 \\
\hline MET 14 & 6.5 & 6.5 & 25.1 & 36.9 & 31.9 & 1.9 & 2.4 & 20.0 & 62.5 & 34.4 & 73.6 \\
\hline ARC39-145-P-2 (5) & 9.0 & 9.1 & 0.1 & 26.7 & 99.6 & 1.0 & 5.3 & 81.1 & 0.0 & 0.0 & 76.3 \\
\hline MET 40 & 2.6 & 2.6 & 32.5 & 42.1 & 22.8 & 2.2 & 2.7 & 15.7 & 100.0 & 49.7 & 56.4 \\
\hline MET 20 & 1.2 & 1.4 & 27.6 & 35.7 & 22.8 & 2.2 & 2.6 & 14.4 & 100.0 & 63.1 & 68.6 \\
\hline MET28 & 4.1 & 5.1 & 28.9 & 43.2 & 33.0 & 2.0 & 2.6 & 20.6 & 62.5 & 56.2 & 76.4 \\
\hline ARC36-2-P-2-54 (2) & 5.7 & 6.9 & 16.9 & 24.9 & 31.9 & 1.4 & 2.6 & 47.2 & 29.2 & 27.2 & 62.5 \\
\hline MET 33 & 6.2 & 4.5 & 26.2 & 35.2 & 25.6 & 1.7 & 2.1 & 21.8 & 79.2 & 35.2 & 66.9 \\
\hline
\end{tabular}




\begin{tabular}{|c|c|c|c|c|c|c|c|c|c|c|c|}
\hline \multirow[t]{2}{*}{ Line name } & \multirow{2}{*}{$\begin{array}{l}\text { Leaf } \\
\text { growth } \\
\text { Stressed }\end{array}$} & \multirow{2}{*}{$\begin{array}{l}\text { Seedling } \\
\text { Color } \\
\text { Stressed } \\
\end{array}$} & \multicolumn{2}{|c|}{$\begin{array}{l}\text { Seedling } \\
\text { height }(\mathrm{cm})\end{array}$} & \multirow[t]{2}{*}{$\begin{array}{l}\text { Reduction } \\
\text { rate }(\%)\end{array}$} & \multicolumn{2}{|c|}{ Tiller number } & \multirow[t]{2}{*}{$\begin{array}{c}\text { Reduction } \\
\text { rate }(\%)\end{array}$} & \multirow{2}{*}{$\begin{array}{l}\text { Survival } \\
\text { rate } \\
\text { Stressed }\end{array}$} & \multicolumn{2}{|c|}{$\begin{array}{l}\text { Grain yield }(\mathrm{g}) / \\
\text { pot }\end{array}$} \\
\hline & & & Stressed & N-stressed & & Stressed & N-stressed & & & Stressed & N-stressed \\
\hline MET 36 & 3.1 & 1.8 & 26.1 & 41.3 & 36.8 & 2.0 & 3.7 & 45.2 & 100.0 & 68.4 & 85.6 \\
\hline NERICA 1 & 1.3 & 2.2 & 29.1 & 35.5 & 18.0 & 2.7 & 3.3 & 17.6 & 100.0 & 85.9 & 97.0 \\
\hline MET 17 & 1.8 & 2.2 & 27.0 & 38.3 & 29.6 & 2.0 & 2.6 & 25.3 & 100.0 & 65.0 & 79.1 \\
\hline MET 7 & 5.8 & 5.8 & 26.4 & 45.0 & 41.4 & 1.3 & 3.2 & 58.6 & 62.5 & 38.4 & 66.5 \\
\hline MET 13 & 5.7 & 6.2 & 22.3 & 38.0 & 41.4 & 1.7 & 2.7 & 35.2 & 58.3 & 46.4 & 87.2 \\
\hline MET 22 & 3.0 & 2.7 & 26.6 & 38.9 & 31.5 & 2.9 & 3.2 & 7.6 & 100.0 & 54.9 & 69.7 \\
\hline WITA 9 & 7.5 & 6.9 & 17.4 & 32.9 & 47.3 & 1.8 & 5.3 & 66.8 & 33.3 & 27.9 & 63.2 \\
\hline MET 60 & 1.3 & 1.7 & 33.1 & 39.6 & 16.4 & 3.0 & 3.3 & 10.8 & 100.0 & 61.7 & 69.4 \\
\hline MET 39 & 2.9 & 2.8 & 23.8 & 36.5 & 34.8 & 2.1 & 3.0 & 29.7 & 100.0 & 46.1 & 57.8 \\
\hline ARC36-2-1-2 & 7.7 & 8.3 & 8.5 & 33.6 & 74.7 & 0.5 & 4.1 & 87.6 & 12.5 & 30.2 & 83.0 \\
\hline MET 31 & 2.9 & 3.0 & 28.6 & 41.6 & 31.2 & 2.6 & 3.5 & 24.0 & 79.2 & 61.5 & 77.6 \\
\hline MET 8 & 3.6 & 4.3 & 22.8 & 38.2 & 40.3 & 2.4 & 3.9 & 37.4 & 79.2 & 46.7 & 90.7 \\
\hline MET 25 & 4.8 & 3.8 & 28.4 & 39.5 & 28.1 & 2.0 & 2.9 & 31.9 & 62.5 & 48.9 & 75.1 \\
\hline MET 35 & 3.9 & 4.1 & 25.0 & 40.3 & 38.0 & 1.4 & 3.1 & 53.8 & 70.8 & 56.8 & 85.4 \\
\hline MET 24 & 2.1 & 2.2 & 26.0 & 39.8 & 34.6 & 2.0 & 2.9 & 31.9 & 100.0 & 56.6 & 73.5 \\
\hline MET23 & 1.1 & 1.0 & 32.1 & 37.1 & 13.7 & 2.2 & 2.9 & 24.0 & 100.0 & 69.0 & 79.0 \\
\hline MET34 & 3.2 & 3.4 & 24.5 & 35.6 & 31.2 & 2.1 & 3.4 & 37.6 & 60.0 & 55.9 & 75.2 \\
\hline MET 18 & 2.5 & 3.4 & 25.2 & 40.3 & 37.4 & 2.3 & 4.6 & 50.8 & 95.9 & 76.3 & 88.1 \\
\hline MET 16 & 2.2 & 3.6 & 28.0 & 42.6 & 34.1 & 1.7 & 2.5 & 34.7 & 100.0 & 49.7 & 61.6 \\
\hline GIZA 177 & 1.0 & 0.7 & 30.2 & 38.0 & 20.5 & 2.8 & 4.3 & 35.3 & 100.0 & 64.8 & 72.0 \\
\hline MET 15 & 4.1 & 6.1 & 27.0 & 39.4 & 31.5 & 2.2 & 2.9 & 24.0 & 62.5 & 50.2 & 76.9 \\
\hline SCRID091-20-2-2-4-4 & 1.2 & 1.4 & 37.2 & 45.0 & 17.3 & 2.6 & 3.3 & 20.3 & 100.0 & 71.8 & 77.5 \\
\hline MET 4 & 3.5 & 3.9 & 23.6 & 38.2 & 38.2 & 1.5 & 3.2 & 52.1 & 79.2 & 38.8 & 58.8 \\
\hline Mean & 4.0 & 4.2 & 23.1 & 38.3 & & 2.0 & 74.3 & & 3.5 & 52.0 & 74.8 \\
\hline LSD (5\%) & 0.9 & 1.2 & 4.6 & 7.5 & & 0.8 & ns & & 16.0 & 29.1 & 87.9 \\
\hline
\end{tabular}


Appendix 2: Tolerance indices of 50 rice genotypes under stress and non-stress condition for grain yield per pot

\begin{tabular}{|c|c|c|c|c|c|c|}
\hline Line name & GY/pot (stressed) & Gy/pot (Non-stressed) & $\%$ Reduction & SSI & TOI & YSI \\
\hline SCRID091-20-2-2-4-4 & 71.8 & 77.5 & 7.3 & 0.2 & 5.7 & 0.9 \\
\hline MET P20 & 63.1 & 68.6 & 8.0 & 0.3 & 5.5 & 0.9 \\
\hline MET P27 & 49.4 & 53.7 & 8.1 & 0.3 & 4.4 & 0.9 \\
\hline MET P5 & 73.0 & 79.6 & 8.2 & 0.3 & 6.5 & 0.9 \\
\hline GIZA 177 & 64.8 & 72.0 & 9.9 & 0.3 & 7.2 & 0.9 \\
\hline MET P60 & 61.8 & 69.4 & 11.0 & 0.4 & 7.6 & 0.9 \\
\hline NERICA 1 & 85.9 & 97.1 & 11.5 & 0.4 & 11.2 & 0.9 \\
\hline MET P40 & 49.7 & 56.4 & 12.0 & 0.4 & 6.7 & 0.9 \\
\hline MET P23 & 69.0 & 79.0 & 12.7 & 0.4 & 10.0 & 0.9 \\
\hline MET P3 & 57.6 & 66.5 & 13.3 & 0.4 & 8.8 & 0.9 \\
\hline MET P18 & 76.3 & 88.1 & 13.4 & 0.4 & 11.8 & 0.9 \\
\hline MET P2 & 63.4 & 74.2 & 14.5 & 0.5 & 10.8 & 0.9 \\
\hline MET P32 & 63.7 & 74.9 & 15.0 & 0.5 & 11.2 & 0.9 \\
\hline MET P37 & 53.9 & 64.1 & 15.9 & 0.5 & 10.2 & 0.8 \\
\hline MET P11 & 72.9 & 88.6 & 17.7 & 0.6 & 15.7 & 0.8 \\
\hline MET P17 & 65.0 & 79.1 & 17.8 & 0.6 & 14.1 & 0.8 \\
\hline MET P9 & 66.3 & 81.4 & 18.5 & 0.6 & 15.1 & 0.8 \\
\hline MET P16 & 49.8 & 61.6 & 19.2 & 0.6 & 11.9 & 0.8 \\
\hline MET P36 & 68.4 & 85.6 & 20.1 & 0.7 & 17.2 & 0.8 \\
\hline MET P39 & 46.1 & 57.8 & 20.3 & 0.7 & 11.7 & 0.8 \\
\hline MET P31 & 61.5 & 77.6 & 20.7 & 0.7 & 16.1 & 0.8 \\
\hline MET P22 & 54.9 & 69.7 & 21.2 & 0.7 & 14.8 & 0.8 \\
\hline MET P24 & 56.6 & 73.5 & 22.9 & 0.8 & 16.9 & 0.8 \\
\hline MET P34 & 55.9 & 75.2 & 25.7 & 0.8 & 19.3 & 0.7 \\
\hline MET P28 & 56.2 & 76.4 & 26.4 & 0.9 & 20.2 & 0.7 \\
\hline MET P29 & 57.4 & 80.9 & 29.1 & 1.0 & 23.5 & 0.7 \\
\hline MET P19 & 51.8 & 74.7 & 30.6 & 1.0 & 22.8 & 0.7 \\
\hline MET P35 & 56.8 & 85.4 & 33.5 & 1.1 & 28.6 & 0.7 \\
\hline MET P4 & 38.8 & 58.8 & 34.1 & 1.1 & 20.1 & 0.7 \\
\hline MET P6 & 44.3 & 67.5 & 34.3 & 1.1 & 23.2 & 0.7 \\
\hline MET P15 & 50.2 & 76.9 & 34.8 & 1.1 & 26.8 & 0.7 \\
\hline MET P25 & 48.9 & 75.1 & 34.9 & 1.2 & 26.2 & 0.7 \\
\hline MET P1 & 43.2 & 73.3 & 41.1 & 1.4 & 30.1 & 0.6 \\
\hline MET P26 & 50.8 & 86.7 & 41.4 & 1.4 & 35.9 & 0.6 \\
\hline MET P7 & 38.4 & 66.5 & 42.3 & 1.4 & 28.1 & 0.6 \\
\hline MET P30 & 38.7 & 68.3 & 43.4 & 1.4 & 29.7 & 0.6 \\
\hline MET P12 & 49.4 & 90.1 & 45.2 & 1.5 & 40.7 & 0.6 \\
\hline MET P21 & 45.8 & 85.7 & 46.6 & 1.5 & 39.9 & 0.5 \\
\hline MET P13 & 46.4 & 87.2 & 46.7 & 1.5 & 40.7 & 0.5 \\
\hline
\end{tabular}




\begin{tabular}{lcccccc} 
MET P33 & 35.2 & 66.9 & 47.4 & 1.6 & 31.7 & 0.5 \\
MET P8 & 46.7 & 90.7 & 48.5 & 1.6 & 44.0 & 0.5 \\
MET P14 & 34.4 & 73.6 & 53.2 & 1.8 & 39.2 & 0.5 \\
WITA 9 & 28.0 & 63.2 & 55.8 & 1.8 & 35.2 & 0.4 \\
ARC36-2-P-2-54 (2) & 27.2 & 62.5 & 56.5 & 1.9 & 35.3 & 0.4 \\
MET P38 & 34.5 & 83.4 & 58.6 & 1.9 & 48.9 & 0.4 \\
MET P10 & 32.8 & 82.6 & 60.3 & 2.0 & 49.8 & 0.4 \\
ARC36-2-1-2 & 30.2 & 83.0 & 63.7 & 2.1 & 52.8 & 0.4 \\
ARC36-4-ET-2(3) & 11.6 & 63.1 & 81.7 & 2.7 & 51.6 & 0.2 \\
ARS126-3-B-1-2(11) & 0.0 & 71.4 & 100.0 & 3.3 & 71.4 & 0.0 \\
ARC39-145-P-2(5) & 0.0 & 76.3 & 100.0 & 3.3 & 76.3 & 0.0 \\
\hline LSD (5\%) & $\mathbf{2 9 . 1}$ & $\mathbf{3 2 . 8}$ & & & & \\
Mean & $\mathbf{5 0 . 0}$ & $\mathbf{7 4 . 8}$ & $\mathbf{3 3 . 1}$ & $\mathbf{1 . 1}$ & $\mathbf{2 4 . 9}$ & $\mathbf{0 . 7}$ \\
Min & $\mathbf{0 . 0}$ & $\mathbf{5 3 . 7}$ & $\mathbf{7 . 3}$ & $\mathbf{0 . 2}$ & $\mathbf{4 . 4}$ & $\mathbf{0 . 0}$ \\
Max & $\mathbf{8 5 . 9}$ & $\mathbf{9 7 . 1}$ & $\mathbf{1 0 0 . 0}$ & $\mathbf{3 . 3}$ & $\mathbf{7 6 . 3}$ & $\mathbf{0 . 9}$ \\
\hline
\end{tabular}

